Journal of Patient-Centered

Volume 2

Issue 3 - Vascular Disease

Article 7

8-14-2015

\title{
Use of Coronary Techniques in Celiac and Hepatic Artery Stenting in Post-Hepatic Transplant Patients
}

Harpreet Parmar

Ryan Beard

Mark W. Mewissen

Armaan Shaikh

Tanvir Bajwa

Follow this and additional works at: https://aah.org/jpcrr

Part of the Cardiology Commons, Cardiovascular Diseases Commons, Cardiovascular System Commons, Diagnosis Commons, Digestive System Diseases Commons, and the Hepatology Commons

\section{Recommended Citation}

Parmar H, Beard R, Mewissen MW, Shaikh A, Bajwa T. Use of coronary techniques in celiac and hepatic artery stenting in post-hepatic transplant patients. J Patient-Centered Res Rev. 2015;2:127-131.

doi:10.17294/2330-0698.1196

Published quarterly by Midwest-based health system Advocate Aurora Health and indexed in PubMed Central, the Journal of Patient-Centered Research and Reviews (JPCRR) is an open access, peer-reviewed medical journal focused on disseminating scholarly works devoted to improving patient-centered care practices, health outcomes, and the patient experience. 


\title{
Use of Coronary Techniques in Celiac and Hepatic Artery Stenting in Post-Hepatic Transplant Patients
}

\author{
Harpreet Parmar, MD, Ryan Beard, MD, Mark W. Mewissen, MD, Armaan Shaikh, DO, Tanvir Bajwa, MD \\ Aurora Cardiovascular Services, Aurora Sinai/Aurora St. Luke's Medical Centers, University of Wisconsin School \\ of Medicine and Public Health, Milwaukee, WI
}

\begin{abstract}
Hepatic artery stenosis (HAS) remains a rare but serious complication after liver transplantation. While invasive surgical techniques were needed for HAS treatment in the past, recently endovascular techniques, including hepatic artery stenting, have been proven to be a safe and effective treatment. The present work focused on a review of the recent literature regarding HAS as well as recent cases demonstrating the various presentations of HAS and the variety of approaches to endovascular intervention. Our single-center experience has shown endovascular treatment of HAS to be safe and effective, including the two specific cases discussed here. While further research is needed, endovascular stenting as a treatment for HAS appears to be a promising, minimally invasive technique to help aid in the long-term health of post-liver transplant patients.
\end{abstract}

Keywords stenosis, liver transplant, hepatic artery, celiac artery

The hepatic artery provides blood flow to both the liver parenchyma and the biliary system and inadequate perfusion may have significant consequences. Hepatic artery stenosis (HAS) is a rare but important complication of orthotopic liver transplantation. The traditional treatment modality formerly consisted of open surgical techniques such as revision of the anastomotic site or placement of a bypass graft. ${ }^{1}$ More recently, however, endovascular techniques have shown to be effective. ${ }^{2,3}$ Herein we review the effectiveness of using traditional coronary artery-based techniques and equipment to treat stenosis of two different abdominal arteries.

\section{CASE DESCRIPTIONS}

\section{Case \#1 - Clinical History}

A 56-year-old woman with a history of autoimmune hepatitis and resultant liver cirrhosis post-orthotopic liver transplant (Model for End-Stage Liver Disease [MELD] score of 28 at time of transplantation) was found on routine laboratory surveillance to have elevated liver enzymes, with an aspartate

Correspondence: Tanvir Bajwa, MD, 2801 W. Kinnickinnic River Parkway, Suite 840, Milwaukee, WI, 53215, T: 414649-3909, F: 414-649-3551, Email: publishing2@aurora.org aminotransferase of 102, alanine aminotransferase of 155 and alkaline phosphatase of 307 . Her liver transplantation was performed 11 months prior and was complicated by postoperative acute hepatic artery thrombosis requiring surgical thrombectomy. As part of her workup, she had Doppler ultrasound of the liver, which came out suspect for HAS and therefore further diagnostic imaging was recommended. She was referred to cardiology, where a hepatic artery angiogram was performed and confirmed HAS.

\section{Procedure}

The left brachial artery was cannulated using a 4-French micropuncture kit and modified Seldinger technique. A 5-French $11-\mathrm{cm}$ sheath was advanced into the artery. Using a 5-French pigtail catheter and J-wire, we obtained access to the descending aorta. The J-wire was removed and a 0.035-inch wire (Supra Core, Abbott Vascular, Abbott Park, IL) was left in the descending aorta. The pigtail catheter and the 5-French sheath were removed and a 6-French $55-\mathrm{cm}$ sheath (Ansel, Cook Medical Inc., Bloomington, IN) was advanced to the abdominal aorta over the Supra Core wire. A 6-French multipurpose guide catheter was then used to selectively engage the celiac trunk and multiple angiographic images obtained. The patient was noted to have a $70-80 \%$ stenosis of hepatic artery 



Figure 1. Angiograms of a 56-year-old woman with hepatic artery stenosis after liver transplantation. A: The patient developed a $70-80 \%$ stenosis at the anastamotic site. B: The lesion was stented with a $4.0 \times 30-\mathrm{mm}$ drug-eluting coronary stent. C: The artery was widely patent after stenting and nitroglycerin administration, with no significant residual stenosis.

at the anastomotic site (Figure 1A). The images were reviewed with the transplant surgeon and the decision was made to proceed with direct stenting of the lesion. The patient was given a weight-based bolus of heparin for anticoagulation to keep the activated clotting time $(\mathrm{ACT})>250 \mathrm{~ms}$. Next, a 0.014-inch wire (Balance Middleweight, Abbott Vascular) was advanced past the hepatic anastomotic site and secured distally. Prior to stenting, balloon angioplasty was performed with a $3.0 \times 20-\mathrm{mm}$ balloon (Trek, Abbott Vascular) at 2 atmospheres for 11 seconds, then at 8 atmospheres for 18 seconds. Percutaneous transluminal angioplasty (PTA) and stenting was performed with a $4.0 \times 30$ $\mathrm{mm}$ drug-eluting stent (Resolute Integrity, Medtronic Inc., Minneapolis, MN) with stent deployment at 9 atmospheres for 6 seconds (Figure 1B). Poststent angiogram revealed a vasospasm, which was relieved with intrahepatic artery nitroglycerin (Figure 1C). The patient tolerated the procedure well without complications. She was transferred to the coronary intensive care unit in a hemodynamically stable condition.

\section{Postprocedure Condition}

Liver enzymes obtained 1 week postprocedure had improved to aspartate aminotransferase of 66, alanine aminotransferase of 104 and alkaline phosphatase of 242 .

\section{Case \#2 - Clinical History}

A 77-year-old woman with history of primary biliary cirrhosis and metastatic hepatocellular carcinoma who underwent previous transcatheter arterial chemoembolization procedures was evaluated the day after orthotopic liver transplant (MELD score of 29 at time of transplantation). A Doppler ultrasound identified suspicious decreased flow velocity indices in the hepatic arteries and its branches as well as a heterogenous $11.1 \times 4.9 \times 7.5-\mathrm{mm}$ fluid collection in Morrison's pouch. Computed tomographic (CT) angiogram revealed a short segment occlusion of the proximal celiac artery with a possible focal dissection. Normal flow was seen in the remainder of the celiac and hepatic arteries. Thus, a celiac artery angiogram was recommended.

\section{Procedure}

The right femoral artery was cannulated using a 4-French micropuncture kit and modified Seldinger technique. A 5-French 11-cm sheath was advanced into the right common femoral artery. A 5-French pigtail catheter was then advanced into the abdominal aorta and a nonselective mesenteric angiogram performed using a power injector (Figure 2A). A dissection within the proximal celiac trunk with $100 \%$ occlusion was identified (Figure 2B). Due to the acute takeoff of the celiac trunk, the decision was made to proceed with intervention via a left brachial artery approach. The left brachial artery was cannulated using a 4-French micropuncture kit and modified Seldinger technique. A 5-French 11-cm sheath was advanced into the artery. The 5-French pigtail catheter and J-wire were used to 
obtain access to the descending aorta. The J-wire was removed and a 0.035-inch Supra Core wire advanced into the descending aorta. Both the 5-French pigtail catheter and $11-\mathrm{cm}$ sheath were then removed. Next, a 6-French 55-cm Ansel sheath was advanced over the Supra Core wire into the descending abdominal aorta. A 6-French multipurpose guide catheter was then used to selectively engage the celiac trunk. The patient was given a weight-based bolus of heparin to keep the ACT $>250$. Next, a 0.014-inch wire (Whisper, Abbott Vascular) was advanced past the celiac occlusion and secured in the splenic artery. Predilation was performed to the multiple segments of the celiac trunk with a $2.0 \times 15-\mathrm{mm}$ balloon (Mini-Trek, Abbott Vascular) at 12 atmospheres for 4 seconds, 9 seconds and 6 seconds. Further predilation was performed in multiple segments of the celiac trunk with a larger 4.0 $\times 20$-mm Trek balloon at 8 atmospheres for 16 seconds and 6 seconds. Subsequent angiogram revealed that a large dissection flap was still visible, thus PTA and stenting was performed to the celiac artery with a 4.0 $\times 22-\mathrm{mm}$ Resolute Integrity drug-eluting stent at 9 atmospheres for 11 seconds. A second $4.0 \times 18-\mathrm{mm}$ Resolute Integrity drug-eluting stent was deployed at 16 atmospheres for 8 seconds inside the first stent to add radial strength (Figure 2C).

\section{Postprocedure Condition}

Final angiogram revealed excellent Thrombolysis in Myocardial Infarction (TIMI) grade 3 flow throughout the celiac trunk and its downstream branches (Figure 2D).

\section{DISCUSSION}

The incidence of hepatic artery stenosis after orthotopic liver transplantation is reported to be approximately 5-13\%. ${ }^{4}$ It most commonly occurs at the anastomotic site between the native and transplanted hepatic arteries. The most common etiologies are thought to be related to operative technique or due to cross-clamp injury. Less frequently, it is associated with allograft rejection, microvascular injury associated with cold preservation injury, or extrinsic compression. ${ }^{2,5}$ Patients who develop HAS postoperatively have approximately a $65 \%$ chance of developing hepatic artery thrombosis, which can be a devastating complication leading to severe biliary damage, acute liver dysfunction and, ultimately, allograft failure. ${ }^{3}$ In addition to HAS, other
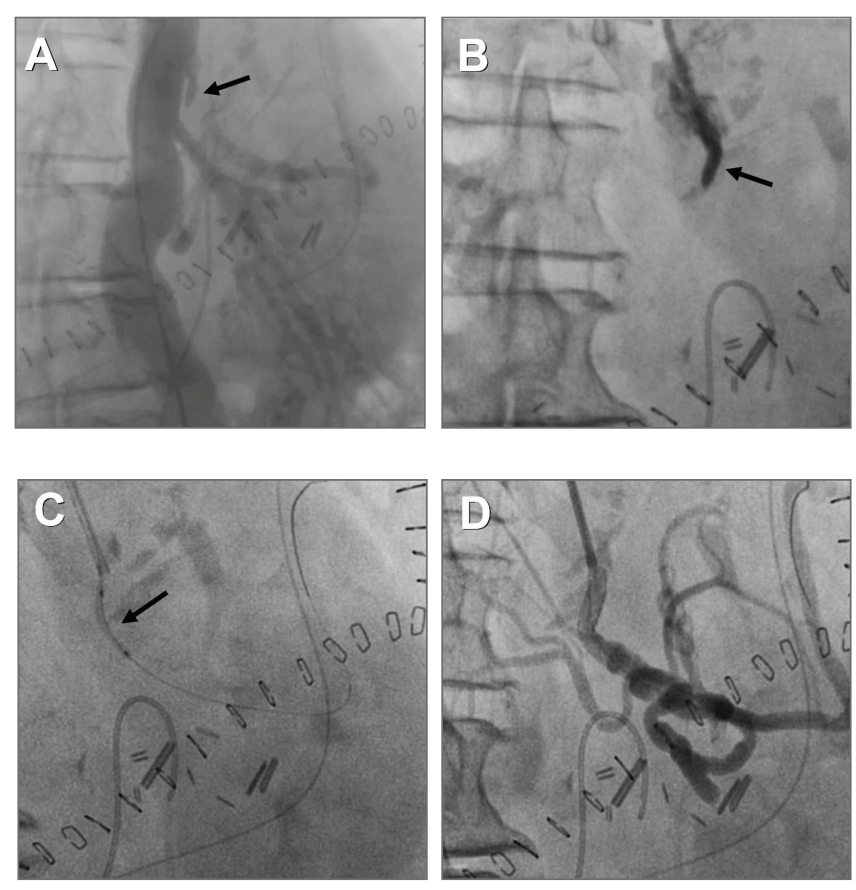

Figure 2. Angiograms of a 77-year-old woman found to have occlusion of celiac artery after liver transplantation. A: Aortogram revealing occlusion of proximal celiac artery. B: Selective angiogram of celiac artery with appearance suggestive of flow-limiting dissection. C: Access to true lumen was obtained, and lesion was stented with $4.0 \times 22-\mathrm{mm}$ and $4.0 \times 18-\mathrm{mm}$ drug-eluting stents. D: Final angiogram showing patent celiac and hepatic artery.

vascular complications that should be assessed include hepatic artery kinking related to excessive vessel length, pseudoaneurysm and arterial dissection.

HAS has been reported to occur most often within the first year after transplantation, with a mean ranging from 100 to 126 days. $^{3}$ Patients may present with elevated liver transaminases or signs of biliary dysfunction such as biliary leak or biliary stricture. ${ }^{2}$ Most often, however, patients are asymptomatic, and HAS is identified incidentally on screening Doppler ultrasound studies. Because of the aggressive nature of the disease, early identification and screening is stressed to prevent potentially irreversible liver damage. ${ }^{1}$ Waiting for a patient to become symptomatic can lead to permanent damage to the allograft and is not the preferred approach. Suspected HAS can be confirmed on CT angiography or traditional catheterbased angiography. 
PTA has been shown to have a technical success rate of $81-93 \%$ in larger HAS trials ${ }^{5}$ and up to $97-100 \%$ in smaller trials. ${ }^{6}$ PTA has been shown to decrease the incidence of hepatic artery thrombosis from $65 \%$ to $19 \%^{3}$ in patients presenting with HAS after transplantation. There is a wide range of variability of primary patency rates after PTA; and although there is no uniformly accepted consensus, there is a trend toward better results with PTA and stenting than with PTA alone. Hamby et al. reported primary patency rates of $83 \%, 74 \%$ and $61 \%$ at 1,3 and 6 months, respectively. ${ }^{6}$ In addition, recent trials have shown long-term patency after PTA of $94 \%$ at 66 months. $^{7}$ Patients undergoing primary stenting required fewer reinterventions than those undergoing PTA alone (31\% vs. $60 \%$ ). If repeat intervention was necessary, the mean time to intervention was 62.5 days in the PTAonly group and 236 days in those treated initially with placement of a stent. ${ }^{6}$ Saad et al. reported primary patency rates at 1 year of $44 \%$ after angioplasty alone, ${ }^{3}$ while Chen et al. demonstrated patency rates of $79 \%$ at 1 year. $^{8}$

A technical issue in endovascular repair, identified in multiple reports, ${ }^{9}$ that may affect procedure success rate and outcome is significant kinking or tortuosity of the hepatic artery. This is most often related to either redundancy from a longer vessel or from significant mismatch in size between donor and recipient arteries. Kinking and tortuosity can greatly increase the technical complexity of the procedure by making it difficult to cross the lesion with a wire or to advance a balloon or stent. Success rates have been reported as low as $14 \%$ when encountering significant arterial kinks. ${ }^{3}$ There is also a trend toward slightly worse patency rates when encountering significant tortuosity. ${ }^{3,6}$ Technical considerations when encountering such anatomy may include the use of a buddy wire, extra stiff wires or a device such as the Quick-Cross ${ }^{\circledR}$ support catheter (Spectranetics Corp., Colorado Springs, CO). ${ }^{3}$

Utilization of coronary-based techniques for treatment of hepatic artery stenosis has been widely reported in the literature. ${ }^{5,6}$ Vessel diameters are often in the range of $2.5-5.0 \mathrm{~mm}$, making coronary equipment a natural choice. Multipurpose coronary guides work well to engage the celiac artery, and 0.014-inch wires with excellent maneuverability work well when encountering severe stenoses or significant tortuosity. Coronary stents are very trackable and have good crossability when encountering these types of lesions. Hamby's authorship group reported poor trackability using self-expanding stents early on in their experience, leading them to change primarily to the use of coronary stents unless there was a large mismatch between the proximal and distal vessel size. ${ }^{6}$

Our two cases of successful treatment of hepatic and celiac artery stenosis after liver transplantation reflect the merits of endovascular approaches. The first patient demonstrated a more classical presentation with elevated liver function tests and was found to have a severe HAS at the anastomotic site. The second patient was found to have a celiac dissection possibly related to the transplant surgery or previous catheterbased chemoembolization procedures. Both patients were successfully treated using traditional coronary equipment including guides, wires and stents. Drugeluting stents were chosen in both cases based on the significantly better patency rates demonstrated when used in the coronary arteries.

\section{CONCLUSIONS}

While more data and understanding is needed to guide therapy and decision-making when encountering hepatic artery stenosis, our experience has been similar to past reports in that angioplasty and stenting proved effective in repairing hepatic and celiac arteries using coronary techniques and equipment.

\section{Patient-Friendly Recap}

- Patients who receive a liver transplant rarely develop blocked arteries in the abdomen.

- This serious complication may be treated using endovascular techniques that are less invasive than open surgery.

- The authors found that hepatic and celiac arteries could be effectively repaired using coronary angioplasty and stenting equipment.

\section{Conflicts of Interest}

None. 


\section{REFERENCES}

1. Stange BJ, Glanemann M, Nuessler NC, Settmacher U, Steinmüller T, Neuhaus P. Hepatic artery thrombosis after adult liver transplantation. Liver Transpl. 2003;9:612-20. CrossRef

2. Ueno $\mathrm{T}$, Jones $\mathrm{G}$, Martin A, et al. Clinical outcomes from hepatic artery stenting in liver transplantation. Liver Transpl. 2006;12:422-7. CrossRef

3. Saad WE, Davies MG, Sahler LG, et al. Hepatic artery stenosis in liver transplant recipients: primary treatment with percutaneous transluminal angioplasty. $J$ Vasc Interv Radiol. 2005;16:795-805. CrossRef

4. Abbasoglu O, Levy MF, Vodapally MS, et al. Hepatic artery stenosis after liver transplantation--incidence, presentation, treatment, and long term outcome. Transplantation. 1997;63:250-5. CrossRef

5. Sabri SS, Saad WE, Schmitt, TM, et al. Endovascular therapy for hepatic artery stenosis and thrombosis following liver transplantation. Vasc Endovascular Surg. 2011;5:447-52. CrossRef

6. Hamby BA, Ramirez DE, Loss GE, et al. Endovascular treatment of hepatic artery stenosis after liver transplantation. $J$ Vasc Surg. 2013;57:1067-72. CrossRef

7. Sommacale D, Aoyagi T, Dondero F, et al. Repeat endovascular treatment of recurring hepatic artery stenoses in orthotopic liver transplantation. Transpl Int. 2013;26:608-15. CrossRef

8. Chen GH, Wang GY, Yang Y, et al. Single-center experience of the therapeutic management of hepatic artery stenosis after orthotopic liver transplantation. Report of 20 cases. Eur Surg Res. 2009;42:21-7. CrossRef

9. Kodama Y, Sakuhara Y, Abo D, et al. Percutaneous transluminal angioplasty for hepatic artery stenosis after living donor liver transplantation. Liver Transpl. 2006;12:465-9. CrossRef

(C) 2015 Aurora Health Care, Inc. 